\title{
A note on global stability of three-dimensional Ricker models
}

\section{Gyllenberg, Mats}

\section{9-01-02}

Gyllenberg , M , Jiang , J \& Niu , L 2019 , ' A note on global stability of three-dimensional Ricker models ' , Journal of Difference Equations and Applications , vol. 25 , no. 1 , pp. 142-150 . https://doi.org/10.1080/10236198.2019.1566459

http://hdl.handle.net/10138/299977

https://doi.org/10.1080/10236198.2019.1566459

acceptedVersion

Downloaded from Helda, University of Helsinki institutional repository.

This is an electronic reprint of the original article.

This reprint may differ from the original in pagination and typographic detail.

Please cite the original version. 


\title{
A note on global stability of three-dimensional Ricker models
}

\author{
Mats Gyllenberg ${ }^{\mathrm{a}}$, Jifa Jiang ${ }^{\mathrm{b}}$ and Lei Niu ${ }^{\mathrm{a}}$ \\ ${ }^{a}$ Department of Mathematics and Statistics, University of Helsinki, Helsinki FIN-00014, \\ Finland; \\ bMathematics and Science College, Shanghai Normal University, Shanghai 200234, China
}

\author{
ARTICLE HISTORY \\ Compiled December 29, 2018
}

\begin{abstract}
In the recent paper [E. C. Balreira, S. Elaydi, and R. Luís, J. Differ. Equ. Appl. 23 (2017), pp. 2037-2071], Balreira, Elaydi and Luís established a good criterion for competitive mappings to have a globally asymptotically stable interior fixed point by a geometric approach. This criterion can be applied to three dimensional Kolmogorov competitive mappings on a monotone region with a carrying simplex whose planar fixed points are saddles but globally asymptotically stable on their positive coordinate planes. For three dimensional Ricker models, they found mild conditions on parameters such that the criterion can be applied to. Observing that Balreira, Elaydi and Luís' discussion is still valid for the monotone region with piecewise smooth boundary, we prove in this note that the interior fixed point of three dimensional Kolmogorov competitive mappings is globally asymptotically stable if they admit a carrying simplex and three planar fixed points which are saddles but globally asymptotically stable on their positive coordinate planes. This result is much easier to apply in the application.
\end{abstract}

\section{KEYWORDS}

Global stability; Ricker model; carrying simplex; global dynamics; phase portrait

\section{Introduction}

The problem on the global dynamics of discrete-time dynamical systems induced by maps is difficult. In particular, how to prove the global stability of a fixed point for discrete-time systems is quite difficult. In the recent article [1], Balreira, Elaydi and Luís have provided a criterion on the global stability of the interior fixed point for the discrete-time competitive system induced by the map

$$
T(x)=\left(x_{1} F_{1}(x), \ldots, x_{n} F_{n}(x)\right)
$$

on $\mathbb{R}_{+}^{n}=[0, \infty)^{n}$, of arbitrary dimension $n$, by using a geometric approach.

Let $\dot{\mathbb{R}}_{+}^{n}:=\left\{x \in \mathbb{R}_{+}^{n}: x_{i}>0, i=1, \ldots, n\right\}$ be the interior of $\mathbb{R}_{+}^{n}$. Denote by $\mathbb{H}_{\{i\}}^{+}$ the $i$ th positive coordinate axis and by $\pi_{i}=\left\{x \in \mathbb{R}_{+}^{n}: x_{i}=0\right\}$ the $i$ th coordinate

Email: mats.gyllenberg@helsinki.fi

Email: jiangjf@shnu.edu.cn

Email: lei.niu@helsinki.fi 
plane. Hereafter, we always assume that each $F_{i}$ in (1) is $C^{1}$ such that $F_{i}(x)>0$ for all $x \in \mathbb{R}_{+}^{n}$. Specifically, Balreira, Elaydi and Luís proved the following global stability criterion in [1].

Theorem 1.1. Consider the map $T: \Omega \subset \mathbb{R}_{+}^{n} \rightarrow \Omega$ given by (1) which admits a unique interior fixed point $p \in \Omega \cap \dot{\mathbb{R}}_{+}^{n}$. Suppose that

(a) $\Omega$ is a monotone region;

(b) $\operatorname{det} D T(x)>0$ for all $x \in \Omega$;

(c) $D T(x)^{-1}>0$ (i.e., all entries of $D T(x)^{-1}$ are positive) for all $x \in \Omega \cap \dot{\mathbb{R}}_{+}^{n}$;

(d) for each $i=1, \ldots, n,\left.T\right|_{\pi_{i}}$, the restriction of $T$ to $\pi_{i}$, has a unique interior fixed point $v_{\{i\}}$ that is globally asymptotically stable in the interior of $\pi_{i}$, but a saddle for $T$;

(e) $T$ admits a carrying simplex $\Sigma \subset \Omega$.

Then $p$ is globally asymptotically stable in $\Omega \cap \dot{\mathbb{R}}_{+}^{n}$.

An open set $\Omega \subset \mathbb{R}_{+}^{n}$ is called a monotone region if $\Gamma=\partial \Omega$ is an oriented smooth hypersurface such that for any $x \in \Gamma \cap \dot{\mathbb{R}}_{+}^{n}$, the normal vector $\eta_{\Gamma}(x)$ at $x$ is strictly positive (see [1]), i.e., $\eta_{\Gamma}(x) \in \dot{\mathbb{R}}_{+}^{n}$, where $\partial \Omega$ is the boundary of $\Omega$ relative to $\mathbb{R}_{+}^{n}$. Here we explore the notation; for a vector (or point) $x \in \mathbb{R}^{n}$, it is said to be positive if $x \in \mathbb{R}_{+}^{n} \backslash\{0\}$ and we write $x>0$; it is said to be strictly positive if $x \in \dot{\mathbb{R}}_{+}^{n}$ and we write $x \gg 0$. Intuitively, a monotone region is a region bounded by the boundary of $\mathbb{R}_{+}^{n}$ and a smooth hypersurface with strictly positive normal. Vacuously, $\mathbb{R}_{+}^{n}$ is a monotone region itself. A carrying simplex for the map $T: \mathbb{R}_{+}^{n} \rightarrow \mathbb{R}_{+}^{n}$ is a compact subset $\Sigma$ of $\mathbb{R}_{+}^{n} \backslash\{0\}$ with the following properties:

(P1) $\Sigma$ is unordered (i.e. if $x, z \in \Sigma$ such that $x_{i} \geq z_{i}$ for all $i=1, \ldots, n$, then $x=z$ );

(P2) $\Sigma$ is homeomorphic to the $(n-1)$-dimensional unit simplex $\Delta^{n-1}=\left\{x \in \mathbb{R}_{+}^{n}\right.$ : $\left.\sum_{i=1}^{n} x_{i}=1\right\}$

(P3) $\forall x \in \mathbb{R}_{+}^{n} \backslash\{0\}$, there is some $z \in \Sigma$ such that $\lim _{k \rightarrow \infty}\left|T^{k}(x)-T^{k}(z)\right|=0$;

(P4) $T(\Sigma)=\Sigma$, and $T: \Sigma \mapsto \Sigma$ is a homeomorphism.

By using their theory (Theorem 1.1), Balreira, Elaydi and Luís [1] further studied the global stability of the three-dimensional Ricker model induced by the map $T$ : $\mathbb{R}_{+}^{3} \rightarrow \mathbb{R}_{+}^{3}$ given by

$$
T_{i}(x)=x_{i} \exp \left(\nu_{i}-\sum_{j=1}^{3} a_{i j} x_{j}\right), \nu_{i}, a_{i j}>0, a_{i i}=1, i, j=1,2,3 .
$$

They proved that the Ricker map $T$ admits a monotone region $\Omega$ and a unique fixed point $p \in \Omega \cap \dot{\mathbb{R}}_{+}^{3}$ such that conditions (b)-(e) in Theorem 1.1 hold on $\Omega$, and hence $p$ is globally asymptotically stable, under the very special condition

$\left(*_{0}\right)$ for all $i, j=1,2,3$ and $j \neq i, \nu_{i}=r, a_{i j}=a$ with $0<r<a<1$ and $a<e^{1-r}-1$.

Recently, the three-dimensional Ricker model (2) was studied by Gyllenberg et al. [8] in detail. They classified all the Ricker maps (2) admitting a carrying simplex under mild conditions (see (4) in Section 2) via the boundary dynamics, and derived a total of 33 stable equivalence classes. It was proved that classes 1-18 which do not admit a positive fixed point have trivial dynamics, i.e. every orbit converges to some fixed point; each map in classes 19-25 admits a unique positive fixed point with index -1 which also 
has trivial dynamics [12]; for classes 26-33, there exists a unique positive fixed point with index 1; moreover, within each of classes 26-31, Neimark-Sacker bifurcations can occur, while in class 32 such bifurcations cannot occur. However, whether nontrivial dynamics (such as Neimark-Sacker bifurcations) can occur in class 33 or not remains an open problem.

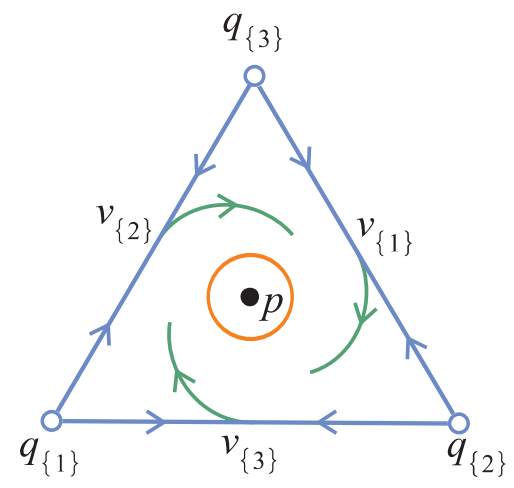

Figure 1. The dynamics on the carrying simplex for Gyllenberg et al.'s class 33 in [8]. For each $i=1,2,3$, $q_{\{i\}}$ is a repelling fixed point lying on $\mathbb{H}_{\{i\}}^{+} ; v_{\{i\}}$ which lies in the interior of $\pi_{i}$ is globally asymptotically stable for $\left.T\right|_{\pi_{i}}$ but a saddle for $T$; there is a unique positive fixed point $p$. The green curves are the unstable manifolds of $v_{\{i\}}, i=1,2,3$, and the big circle $\bigcirc$ in the interior denotes a region of unknown dynamics.

We notice that the Ricker map (2) satisfies the conditions (d) and (e) in Theorem 1.1 if it is in Gyllenberg et al.'s class 33, i.e., each map $T$ in this class admits a carrying simplex and in the interior of each $\pi_{i}$, there exists a fixed point, say $v_{\{i\}}$, which is globally asymptotically stable for $T_{\pi_{i}}$, but a saddle for $T$; see Fig. 1. Moreover, each Ricker map (2) in this class satisfies the conditions (b) and (c) on a forward invariant hyperrectangle $[0, q]:=\left\{x \in \mathbb{R}^{3}: 0 \leq x_{i} \leq q_{i}, i=1,2,3\right\}$ (i.e., $T([0, q]) \subset[0, q]$ ) which contains the carrying simplex for some $q=\left(q_{1}, q_{2}, q_{3}\right)^{\tau} \gg 0$. These facts suggest that the positive fixed point in the class 33 might be globally asymptotically stable such that nontrivial dynamics cannot occur in this class by Theorem 1.1. Unfortunately, since the boundary of $[0, q]$ in $\mathbb{R}_{+}^{3}$ is not smooth such that it has a strictly positive normal vector at every point, i.e., $[0, q]$ is not a monotone region, one cannot apply Theorem 1.1 directly to such Ricker maps restricted to $[0, q]$.

Indeed, in order to apply Theorem 1.1 to discrete-time models, one must find a forward invariant and globally attracting set, say $\Omega$, which has a smooth boundary with strictly positive normal vector at every point, i.e. $T(\Omega) \subset \Omega$ and $\Omega$ is a monotone region. However, it is usually not easy to construct such a set for concrete discrete-time models (see [14, 15]), where techniques and complicated calculation are often required. Recall that in [1], Balreira, Elaydi and Luís only proved that $\Omega$ is a globally attracting set for the Ricker map (2) under the special parameter conditions

$\left(*_{1}\right)$ for all $i, j=1,2,3,0 \leq \nu_{i} \leq a_{i j}$;

$\left(*_{2}\right)$ the parameters satisfy $a_{12} a_{23} a_{31}=a_{13} a_{32} a_{21}$,

where $\Omega$ is the region containing the origin bounded by the component of $L C_{-1}=$ $\left\{x \in \mathbb{R}_{+}^{3}: \operatorname{det} D T(x)=0\right\}$. By a long computation in [1, Appendix 2] (more than two pages), they derived that the normal vector at every point on the boundary of $\Omega$ is strictly positive, and proved that $\Omega$ is globally attracting in [1, Lemma 5.1] (almost one page) under conditions $\left(*_{1}\right)$ and $\left(*_{2}\right)$. On the other hand, it seems not easy to show that such $\Omega$ is a forward invariant monotone region when $\left(*_{1}\right)$ and $\left(*_{2}\right)$ do not 
hold. Due to this difficulty, Theorem 1.1 does not seem to apply easily to concrete discrete-time models, such as the Ricker model (2) with much more general parameter conditions.

However, for most competitive discrete-time models on $\mathbb{R}_{+}^{n}$, one can usually find a forward invariant and globally attracting hyperrectangle $[0, u]$ with $u \gg 0$ (see [9]), such as the Ricker map (2) (see [8]). Thus, in this note, we try to study the global stability of the Ricker map (2) in Gyllenberg et al.'s class 33 restricted to a forward invariant hyperrectangle directly without constructing a monotone region by the method in [1]. In fact, we prove that Balreira, Elaydi and Luís's result in [1] is still valid for the map $T:[0, u] \rightarrow[0, u]$ which satisfies conditions (b)-(e) in Theorem 1.1 on $\Omega=[0, u]$ by following their idea. Therefore, in this note we provide the following weaker version (Theorem 1.2) of Balreira, Elaydi and Luís's theorem, which can be applied to the Ricker map (2) easily, and we prove that the positive fixed point is indeed globally asymptotically stable for each Ricker map (2) in Gyllenberg et al.'s class 33, i.e. the positive fixed point is globally asymptotically stable for every Ricker map admitting a carrying simplex with boundary dynamics shown in Fig. 1. As a consequence, we can describe the global dynamics of Gyllenberg et al.'s class 33 clearly, and the phase portrait on the carrying simplex for this class is shown in Fig. 2.

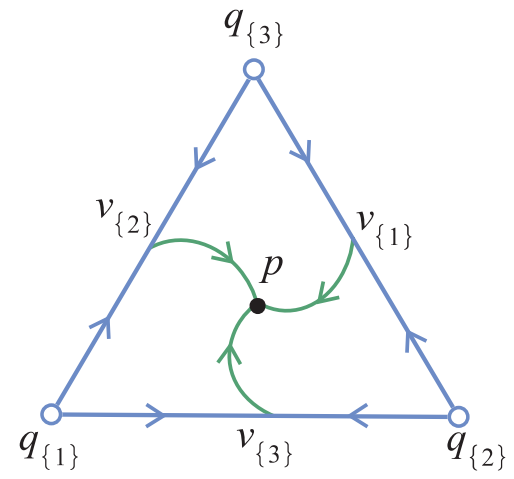

Figure 2. The global dynamics on $\Sigma$ of Gyllenberg et al.'s class 33 . The interior fixed point $p$ is globally asymptotically stable.

Theorem 1.2. Consider the map $T:[0, u] \rightarrow[0, u]$ for some $u \gg 0$ given by (1) which admits a unique interior fixed point $p \in[0, u] \cap \dot{\mathbb{R}}_{+}^{n}$. Assume that

(a) $\operatorname{det} D T(x)>0$ for all $x \in[0, u]$;

(b) $D T(x)^{-1}>0$ for all $x \in[0, u] \cap \dot{\mathbb{R}}_{+}^{n}$;

(c) for each $i=1, \ldots, n,\left.T\right|_{\pi_{i}}$ has a unique interior fixed point $v_{\{i\}}$ that is globally asymptotically stable in the interior of $\pi_{i}$, but a saddle for $T$;

(d) $T$ admits a carrying simplex $\Sigma \subset[0, u]$;

Then $p$ is globally asymptotically stable in $[0, u] \cap \dot{\mathbb{R}}_{+}^{n}$.

Remark 1. Since $\operatorname{det} D T(x)>0$ for all $x \in[0, u], T$ is a local homeomorphism on $[0, u]$. Recall that $F_{i}(x)>0$ for all $x \in \mathbb{R}_{+}^{n}, i=1, \ldots, n$, so one has $T_{i}(x)>0 \Leftrightarrow x_{i}>0$ and in particular, $T^{-1}(\{0\})=\{0\}$. It then follows from Lemma 3.4 in [2, p. 27] that $T$ is actually a homeomorphism from $[0, u]$ onto its image because $[0, u]$ is a compact set (see also Lemma 4.1 in [13]). Conditions (a) and (b) imply the cofactors of the Jacobian matrix $D T(x)$ are positive for all $x \in[0, u] \cap \dot{\mathbb{R}}_{+}^{n}$. Denote by $C_{i j}(D T(x))$ the $(i, j)$-cofactor of $D T(x)$. Let $A=[0, u]$. Note that the boundary of $A$ relative to $\mathbb{R}_{+}^{n}$ is 
piecewise smooth. For any $x \in A \cap \dot{\mathbb{R}}_{+}^{n}$, one can choose a piecewise smooth hypersurface $\Gamma$ containing $x$ such that for each smooth piece, the normal vector at every point is positive. Indeed, $\Gamma$ can be chosen to be the boundary of a ball at the origin or a translation of the boundary of $A$. Suppose $\Gamma=\cup_{i=1}^{m} C_{i}, 1 \leq m<+\infty$, where $C_{i}$ is the $i$ th smooth piece of $\Gamma$. For every $x \in C_{i} \cap \dot{\mathbb{R}}_{+}^{n}$, one has the normal $\eta_{C_{i}}(x)>0$. Let $S_{i}=T\left(C_{i}\right)$. Then $T(\Gamma)=\cup_{i=1}^{m} S_{i}$ is also a piecewise smooth hypersurface in $A$ such that $T(x) \in T(\Gamma)$. It then follows from [1, Equ. (8)] that

$$
\left(\eta_{S_{k}}\right)_{i}=\left(\eta_{T\left(C_{k}\right)}\right)_{i}=\sum_{j=1}^{n} C_{i j}(D T(x)) \cdot\left(\eta_{C_{k}}\right)_{j}>0,
$$

because each cofactor $C_{i j}(D T(x))$ is positive and at least one $\left(\eta_{C_{k}}\right)_{j}>0$. This implies that $\eta_{S_{i}}(T(x)) \gg 0$, i.e. $T(\Gamma) \subset A$ is a piecewise smooth hypersurface such that for each smooth piece $S_{i}$, the normal vector at every point is strictly positive. Now one can just repeat the proof in [1] to obtain Theorem 1.2.

Theorem 1.2 is easier to verify than Theorem 1.1 in the application. In particular, one can apply Theorem 1.2 to the map $T$ given by (1) directly after proving the existence of a carrying simplex (by Lemma 2.1 below). In Section 2, we show that one can easily apply Theorem 1.2 to the Ricker model (2) without complicated calculations.

\section{Global stability of the Ricker model}

Before we study the global stability of the Ricker model (2), we first recall a criterion provided in [11] on the existence of a carrying simplex for the map $T$ given by (1).

Lemma 2.1 (Existence Criterion of Carrying Simplex [11]). Assume the map T given by (1) satisfies that

$\Upsilon 1) \partial F_{i}(x) / \partial x_{j}<0$ holds for all $x \in \mathbb{R}_{+}^{n}$ and $i, j=1, \ldots, n$;

$\Upsilon 2)$ For each $i=1, \ldots, n,\left.T\right|_{\mathbb{H}_{\{i\}}^{+}}: \mathbb{H}_{\{i\}}^{+} \rightarrow \mathbb{H}_{\{i\}}^{+}$has a fixed point $q_{\{i\}}=q_{i} e_{\{i\}}$ with $q_{i}>0$, where $e_{\{i\}}$ is the unit vector pointing in the ith direction;

$\Upsilon 3) \forall x \in[0, q] \backslash\{0\}, F_{i}(x)+\sum_{j \in \kappa(x)} x_{j} \frac{\partial F_{i}(x)}{\partial x_{j}}>0$ holds for any $i \in \kappa(x)\left(\right.$ or $F_{i}(x)+$ $\sum_{j \in \kappa(x)} x_{i} \frac{\partial F_{i}(x)}{\partial x_{j}}>0$ holds for any $\left.i \in \kappa(x)\right)$, where $q=\left(q_{1}, \ldots, q_{n}\right)^{\tau}$ and $\kappa(x)=$ $\left\{j: x_{j}>0\right\}$ is the support of $x$.

Then $[0, q]$ is forward invariant under $T$, and $T$ has a carrying simplex $\Sigma \subset[0, q]$.

Note that $\Upsilon 3)$ implies that $\operatorname{det} D T(x)>0$ for all $x \in[0, q]$, and together with $\Upsilon 1)$ it guarantees $\left(D T(x)_{\kappa(x)}\right)^{-1}>0$ for all $x \in[0, q] \backslash\{0\}$; see [7, 11]. Therefore, $T:[0, q] \rightarrow[0, q]$ in Lemma 2.1 satisfies (a), (b) and (d) in Theorem 1.2.

We are now ready to study the global stability of the Ricker model (2). For convenience, we rewrite the Ricker model (2) into the following form

$$
T_{i}(x)=x_{i} \exp \left(\nu_{i}\left(1-\sum_{j=1}^{3} \mu_{i j} x_{j}\right)\right), \nu_{i}, \mu_{i j}>0, i, j=1,2,3 .
$$


Assume that the parameters satisfy

$$
\nu_{i}<\mu_{i i} / \sum_{j=1}^{3} \mu_{i j}, \text { or } \nu_{i}<1 /\left(\sum_{j=1}^{3} \frac{\mu_{i j}}{\mu_{j j}}\right), i=1,2,3 .
$$

Denote by

$$
\mathrm{CRC}(3):=\left\{T \in \mathcal{T}\left(\mathbb{R}_{+}^{3}\right): T_{i}(x)=x_{i} \exp \left(\nu_{i}\left(1-\sum_{j=1}^{3} \mu_{i j} x_{j}\right)\right), \nu_{i}, \mu_{i j}>0,(4) \text { holds }\right\}
$$

the set of Ricker maps (3) satisfying (4), where $\mathcal{T}\left(\mathbb{R}_{+}^{3}\right)$ denotes the set of all maps taking $\mathbb{R}_{+}^{3}$ into itself. It is easy to check that the Ricker map (3) satisfies $\Upsilon 3$ ) in Lemma 2.1 if condition (4) holds, and hence each Ricker map in CRC(3) admits a carrying simplex $\Sigma \subset[0, q]$ because $\Upsilon 1)$ and $\Upsilon 2)$ in Lemma 2.1 hold for each Ricker map (3), where $q=\left(q_{1}, q_{2}, q_{3}\right)^{\tau}$ with $q_{i}=\frac{1}{\mu_{i i}}$. Moreover, Gyllenberg et al. classified CRC(3) via the dynamics on the boundary of the carrying simplex in [8], and they derived a total of 33 stable equivalence classes which are described in terms of inequalities on the parameters $\nu_{i}$ and $\mu_{i j}, i, j=1,2,3$. Since each Ricker map $T \in \mathrm{CRC}(3)$ satisfies conditions $\Upsilon 1$ )- $\Upsilon 3$ ) in Lemma 2.1, we know that $T:[0, q] \rightarrow[0, q]$ satisfies (a), (b) and (d) in Theorem 1.2. According to [8], we have the following Lemma 2.2. We refer the reader to [8] for the detailed proof of this lemma, and we only state it here.

Lemma $2.2([8])$. The Ricker map $T \in \mathrm{CRC}(3)$ is in class 33 if the parameters $\nu_{i}, \mu_{i j}$ satisfy the condition (4) and the following inequalities

(i) $\gamma_{12}>0, \gamma_{13}>0, \gamma_{21}>0, \gamma_{23}>0, \gamma_{31}>0, \gamma_{32}>0$;

(ii) $\mu_{12} \beta_{23}+\mu_{13} \beta_{32}<1$;

(iii) $\mu_{21} \beta_{13}+\mu_{23} \beta_{31}<1$;

(iv) $\mu_{31} \beta_{12}+\mu_{32} \beta_{21}<1$,

where $\gamma_{i j}=\mu_{i i}-\mu_{j i}$ and $\beta_{i j}=\frac{\mu_{j j}-\mu_{i j}}{\mu_{i i} \mu_{i j}-\mu_{i j} \mu_{j i}}, i, j=1,2,3$ and $i \neq j$. In this class, the map $T$ has three nontrivial hyperbolic axial fixed points $q_{\{1\}}, q_{\{2\}}$ and $q_{\{3\}}$ with $q_{\{i\}} \in \mathbb{H}_{\{i\}}^{+}$, three hyperbolic planar fixed points $v_{\{1\}}, v_{\{2\}}$ and $v_{\{3\}}$ with $v_{\{i\}}$ lying on the interior of $\pi_{i}$, and a unique interior fixed point $p \in \dot{\mathbb{R}}_{+}^{3}$. For all $i=1,2,3$, $v_{\{i\}}$ is globally asymptotically stable for $T_{\pi_{i}}$, but a saddle for $T$. Moreover, each $q_{\{i\}}$ is a repeller on $\Sigma$ and $v_{\{i\}}$ is a saddle on $\Sigma, i=1,2,3$. The phase portrait on $\Sigma$ is shown in Fig. 1.

The conditions (i)-(iv) in Lemma 2.2 have clear biological interpretations, which we present here. The condition $\gamma_{i j}>0$ means that species type $j$ can invade the monomorphic population of species type $i$ at the steady state $q_{\{i\}}$ for $j \neq i$. Furthermore, condition (i) implies that the two species types $i$ and $j$ will attain a coexistence steady state $v_{\{k\}}$ in the absence of species type $k$; here $i, j, k$ are distinct. Condition $\mu_{k i} \beta_{i j}+\mu_{k j} \beta_{j i}<1$ means that species type $k$ can invade the dimorphic population set by species types $i$ and $j$ at the steady state $v_{\{k\}}$. For a discussion of these notions and their consequences for evolutionary dynamics, we refer the reader to [3-6].

Gyllenberg-Jiang-Niu-Yan's open problem ([8]). Whether there is a NeimarkSacker bifurcation in class 33 for Ricker models (3)? Whether the interior fixed point in class 33 is globally asymptotically stable? 
Theorem 2.3. The interior fixed point $p$ is globally asymptotically stable in $\dot{\mathbb{R}}_{+}^{3}$ for each Ricker map $T \in \mathrm{CRC}(3)$ in the class 33. The global dynamics on $\Sigma$ is shown in Fig. 2.

Proof. By Lemma 2.2, each Ricker map $T$ in the class 33 has a unique interior fixed point $p \in[0, q] \cap \dot{\mathbb{R}}_{+}^{3}$, and three planar fixed points $v_{\{1\}}, v_{\{2\}}$ and $v_{\{3\}}$ with $v_{\{i\}}$ lying on the interior of $\pi_{i}$ such that $v_{\{i\}}$ is globally asymptotically stable for $T_{\pi_{i}}$ but a saddle for $T, i=1,2,3$. Therefore, each $T$ in the class 33 satisfies condition (c) in Theorem 1.2. Recall that $T:[0, q] \rightarrow[0, q]$ satisfies (a), (b) and (d) in Theorem 1.2 , so $p$ is globally asymptotically stable in $[0, q] \cap \dot{\mathbb{R}}_{+}^{3}$ by Theorem 1.2 . In particular, $T^{k}(z) \rightarrow p$ as $k \rightarrow+\infty$ for all $z \in \Sigma \cap \dot{\mathbb{R}}_{+}^{3}$ because $\Sigma \subset[0, q]$, and hence every orbit on $\Sigma$ tends to a nontrivial fixed point of $T$ by Lemma 2.2; see Fig. 2 for the global dynamics on $\Sigma$. It then follows from the property (P3) of $\Sigma$ that every orbit in $\mathbb{R}_{+}^{3}$ tends to a nontrivial fixed point. We now show that $T^{k}(x) \rightarrow p$ as $k \rightarrow+\infty$ for all $x \in \dot{\mathbb{R}}_{+}^{3}$. According to Lemma 2.2, one has

$$
W^{s}\left(x^{*}\right) \cap \dot{\mathbb{R}}_{+}^{3}=\emptyset
$$

for each nontrivial boundary fixed point $x^{*} \in\left\{q_{\{1\}}, q_{\{2\}}, q_{\{3\}}, v_{\{1\}}, v_{\{2\}}, v_{\{3\}}\right\}$, where $W^{s}\left(x^{*}\right)$ is the stable manifold of $x^{*}$, because $q_{\{i\}}$ and $v_{\{i\}}$ are hyperbolic, $i=1,2,3$. We conclude that $T^{k}(x) \rightarrow p$ as $k \rightarrow+\infty$ for all $x \in \dot{\mathbb{R}}_{+}^{3}$. This completes the proof.

Consider the special case of the three-dimensional Ricker model (2) with $\nu_{i}=r>$ $0, a_{i i}=1$ and $a_{i j}=a>0$ for all $i, j=1,2,3$ and $j \neq i$, that is,

$$
T(x)=\left(x_{1} e^{r-x_{1}-a x_{2}-a x_{3}}, x_{2} e^{r-a x_{1}-x_{2}-a x_{3}}, x_{3} e^{r-a x_{1}-a x_{2}-x_{3}}\right) .
$$

Ricker model (5) can be written as

$$
T(x)=\left(x_{1} e^{r\left(1-\frac{1}{r} x_{1}-\frac{a}{r} x_{2}-\frac{a}{r} x_{3}\right)}, x_{2} e^{r\left(1-\frac{a}{r} x_{1}-\frac{1}{r} x_{2}-\frac{a}{r} x_{3}\right)}, x_{3} e^{r\left(1-\frac{a}{r} x_{1}-\frac{a}{r} x_{2}-\frac{1}{r} x_{3}\right)}\right) .
$$

Let $\mu_{i i}=\frac{1}{r}$ and $\mu_{i j}=\frac{a}{r}, i, j=1,2,3$ and $j \neq i$. As far as we have known, the existing criterion of the carrying simplex for $(5)$ is $0<r<\frac{1}{1+2 a}$ (see $[10,11,13]$ ). It seems to us that Balreira, Elaydi and Luís [1] did not give the parameter conditions to guarantee that the condition (e) in Theorem 1.1 holds. In addition, when $a<1$, the conditions (i)-(iv) in Lemma 2.2 hold and hence the Ricker model (5) is in the class 33. In this case, $T$ has three nontrivial hyperbolic axial fixed points $q_{\{1\}}=(r, 0,0)$, $q_{\{2\}}=(0, r, 0), q_{\{3\}}=(0,0, r)$, three hyperbolic planar fixed points

$$
v_{\{1\}}=\left(0, \frac{r}{1+a}, \frac{r}{1+a}\right), v_{\{2\}}=\left(\frac{r}{1+a}, 0, \frac{r}{1+a}\right), v_{\{3\}}=\left(\frac{r}{1+a}, \frac{r}{1+a}, 0\right),
$$

and a unique interior fixed point

$$
p=\left(\frac{r}{1+2 a}, \frac{r}{1+2 a}, \frac{r}{1+2 a}\right) .
$$

By Lemma 2.2, $v_{\{i\}}$ is globally asymptotically stable in the interior of $\pi_{i}$, but a saddle for $T$. Therefore, it follows from Theorem 2.3 that the Ricker model (5) has a globally asymptotically stable interior fixed point when $0<r<\frac{1}{1+2 a}$ and $0<a<1$. 
Theorem 2.3 solves Gyllenberg-Jiang-Niu-Yan's open problem on class 33 for the Ricker model (3) in [8]. That is, the interior fixed point in Gyllenberg et al.'s class 33 is globally asymptotically stable, and hence nontrivial dynamics (such as NeimarkSacker bifurcations) cannot occur in this class for the Ricker model (3). Biologically, Theorem 2.3 means that for three competing species modeled by the Ricker model (3) under condition (4), if each species can invade either of the other two species, and also the coexistence steady state of the other two species, then there will be coexistence in the form of a globally asymptotically stable positive fixed point.

Remark 2. If the set $[0, u]$ in Theorem 1.2 is replaced by $[0, \infty)^{n}$, then Theorem 1.2 is the same as Theorem 1.1. However, in application, if one has proved the existence of the carrying simplex by Lemma 2.1, then one can apply Theorem 1.2 directly to $\left.T\right|_{[0, q]}$ just as Theorem 2.3 because $\Sigma \subset[0, q]$ and $T:[0, q] \rightarrow[0, q]$ satisfies conditions (a), (b) and (d) in Theorem 1.2. In other words, conditions $\Upsilon 1)-\Upsilon 3$ ) in Lemma 2.1 and condition c) in Theorem 1.2 imply the global stability of $p$ provided $p$ is the unique interior fixed point in $\dot{\mathbb{R}}_{+}^{n}$. For example, one can apply Theorem 1.2 directly to the classical 3D Leslie-Gower model [11]

$$
T_{i}(x)=\frac{\left(1+c_{i}\right) x_{i}}{1+\sum_{j=1}^{3} b_{i j} x_{j}}, c_{i}, b_{i j}>0, i, j=1,2,3
$$

and the Atkinson-Allen model [7]

$$
T_{i}(x)=\frac{\left(1+c_{i}\right)\left(1-a_{i}\right) x_{i}}{1+\sum_{j=1}^{3} b_{i j} x_{j}}+a_{i} x_{i}, 0<a_{i}<1, c_{i}, b_{i j}>0, i, j=1,2,3 .
$$

Note that conditions $\Upsilon 1)-\Upsilon 3$ ) in Lemma 2.1 and condition c) in Theorem 1.2 hold for the class 33 of the Leslie-Gower model (7) and the Atkinson-Allen model (8) in [11] and [7] respectively, so the unique interior fixed point in class 33 for these two models is globally asymptotically stable. See Fig. 2 for the global dynamics on the carrying simplex for this class.

Finally, we point out that if one use the criteria of Hirsch [10] or [13] for the existence of carrying simplex to (7) and (8), one should add additional parameter restriction conditions. However, when the criterion stated in Lemma 2.1 is applied to Leslie-Gower model (7) and the Atkinson-Allen model (8), there is no parameter restrictions (see [11] for details).

\section{Acknowledgements}

The authors are very grateful to Prof. Saber Elaydi, Prof. Janusz Mierczyński and Dr. Stephen Baigent for valuable suggestions and also thank Prof. Saber Elaydi for sending us the manuscript of reference [1].

\section{Funding}

This work is supported by the National Natural Science Foundation of China (NSFC) under Grant No. 11771295, Shanghai Gaofeng Project for University Academic Program Development, and the Academy of Finland. 


\section{References}

[1] E. C. Balreira, S. Elaydi, and R. Luís, Global stability of higher dimensional monotone maps, J. Differ. Equ. Appl. 23 (2017), pp. 2037-2071.

[2] S. N. Chow and J. K. Hale, Methods of Bifurcation Theory, Springer-Verlag, New York, 1982.

[3] S. A. H. Geritz, Resident-invader dynamics and the coexistence of similar strategies, J. Math. Biol., 50 (2005), pp. 67-82.

[4] S. A. H. Geritz, M. Gyllenberg, F. J. A. Jacobs and K. Parvinen, Invasion dynamics and attractor inheritance, J. Math. Biol., 44 (2002), pp. 548-560.

[5] S. A. H. Geritz, E. Kisdi, G. Meszéna and J. A. J. Metz, Evolutionarily singular strategies and the adaptive growth and branching of the evolutionary tree, Evolutionary Ecology, 12 (1998), pp. 35-57.

[6] S. A. H. Geritz, J. A. J. Metz, E. Kisdi and G. Meszéna, Dynamics of adaptation and evolutionary branching, Phys. Rev. Letters, 78 (1997), pp. 2024-2027.

[7] M. Gyllenberg, J. Jiang, L. Niu, and P. Yan, On the classification of generalized competitive Atkinson-Allen models via the dynamics on the boundary of the carrying simplex, Discrete Contin. Dyn. Syst., 38 (2018), pp. 615-650.

[8] M. Gyllenberg, J. Jiang, L. Niu, and P. Yan, On the dynamics of multi-species Ricker models admitting a carrying simplex, submitted.

[9] M. Gyllenberg, J. Jiang, L. Niu, and P. Yan, Permanence and universal classification for discrete-time competitive systems via the carrying simplex, submitted.

[10] M. W. Hirsch, On existence and uniqueness of the carrying simplex for competitive $d y$ namical systems, J. Biol. Dyn., 2 (2008), pp. 169-179.

[11] J. Jiang and L. Niu, On the equivalent classification of three-dimensional competitive Leslie/Gower models via the boundary dynamics on the carrying simplex, J. Math. Biol. 74 (2017), pp. 1223-1261.

[12] L. Niu and A. Ruiz-Herrera, Trivial dynamics in discrete-time systems: carrying simplex and translation arcs, Nonlinearity 31 (2018), pp. 2633-2650.

[13] A. Ruiz-Herrera, Exclusion and dominance in discrete population models via the carrying simplex, J. Differ. Equ. Appl. 19 (2013), pp. 96-113.

[14] R. J. Sacker, A Note: An invariance theorem for mappings II, J. Dyn. Diff. Equat. 24 (2012), pp. 595-599.

[15] R. J. Sacker, Short note: An invariance theorem for mappings, J. Differ. Equ. Appl. 18 (2012), pp. 163-166. 